\title{
Observations of Hot-Jupiter occultations combining Spitzer and Kepler photometry
}

\author{
J. M. Désert ${ }^{1}$, D. Charbonneau ${ }^{1}$, N. Madhusudhan ${ }^{2}$, J. Fortney ${ }^{3}$,
}

F. Fressin ${ }^{1}$, H. Knutson ${ }^{4}$, D. Deming ${ }^{5} \&$ the Kepler Team

${ }^{1}$ Harvard-Smithsonian Center for Astrophysics, 60 Garden Street, Cambridge, MA02138 [jdesert@cfa.harvard.edu]

${ }^{2}$ Department of Astrophysical Sciences, Princeton University,Princeton, NJ 05844

${ }^{3}$ Department of Astronomy and Astrophysics, University of California at Santa Cruz, Santa Cruz, CA 95064

${ }^{4}$ Department of Astronomy, University of California, Berkeley, CA 94720-3411, $U S A$

${ }^{5}$ Planetary Systems Laboratory, NASA?s Goddard Space Flight Center, Greenbelt $M D 20771$

\begin{abstract}
We present the status of an ongoing program which aim at measuring occultations by their parent stars of transiting hot giant exoplanets discovered recently by Kepler. The observations are obtained in the near infrared with WarmSpitzer Space Telescope and at optical wavelengths by combining more than a year of Kepler photometry. The investigation consists of measuring the mid-occultation times and the relative occultation depths in each band-passes. Our measurements of occultations depths in the Kepler bandpass is turned into the determination of the optical geometric albedo $A_{g}$ in this wavelength domain. The brightness temperatures of these planets are deduced from the infrared observations. We combine the optical and near infrared planetary emergent fluxes to obtain broad band emergent spectra of individual planet. We finally compare these spectra to hot Jupiter atmospheric models in order broadly distinguishing these atmospheres between different classes of models.
\end{abstract}

\section{Introduction}

The highly irradiated transiting hot-Jupiter currently provide the best opportunities for studying exoplanetary atmospheres in emission, during planetary occultations, when the exoplanets pass behind their parent stars. In this configuration, the planet-to-star flux ratio can be observationally measured. The planet-to-star flux ratio is ten times higher at infrared wavelengths, due to the thermal emission from the planet, compared to optical wavelengths, more sensitive to reflectance (Fortney et al. 2005 ,Seager et al. 2005). Consequently, only few objects may be observed in the visible, those for which we will have enough observations to combine during occultations to improve the signal-to-noise and reveal the eclipse event. However, these objects are very valuable

This is an Open Access article distributed under the terms of the Creative Commons Attribution-Noncommercial License 3.0, which permits unrestricted use, distribution, and reproduction in any noncommercial medium, provided the original work is properly cited. 
for understanding the energy budget of VHJs and, to some extent, for comparative exoplanetology. In particular, the VHJs detected by CoRoT and Kepler are interesting planets to study their atmosphere since the multiple occultations gathered in the optical bandpass from these space telescopes staring at these targets can be combined to reveal them (Borucki et al. 2009).

In this work, we combine occultation measurements obtained in the optical with Kepler and in the infrared with Warm-Spitzer to learn about the atmospheric properties of two VHJs, Kepler-5b (Koch et al. 2010) and Kepler-6b (Dunham et al. 2010), discovered using Kepler spacecraft. Interestingly, the photometry of the host stars are continuously monitored by the Kepler space telescope, allowing us to gather more than a year of observations and combine multiple occultations to improve the signal-to-noise. These two planets have masses and radii which are common amongst the known transiting VHJs (Koch et al. 2010, Dunham et al. 2010, Latham et al. 2010). Therefore, they are good candidates to study especially if we want to generalize our conclusions for comparative exoplanetology. We also obtained measurements of these targets in the near infrared with the Warm-Spitzer space telescope during occultations. Measurements of the optical and near infrared emergent fluxes from the planet allows us to derive the Bond albedo of these objects, to learn about the energy distribution and to address whether or not an atmospheric thermal inversion can be revealed. Because our infrared measurements are near the peak of VHJ's spectral energy distribution, our optical measurements are mainly due to thermal radiation rather than purely reflected light.

\section{Warm-Spitzer observations and photometry of Kepler-5b and Kepler-6b}

As Spitzer has exhausted its cryogen of liquid coolant on 15 May 2009, only the two first channels, at 3.6 (channel 1) and $4.5 \mu \mathrm{m}$ (channel 2), of the Infrared Array Camera (IRAC; are available in the post-cryogenic mission. Kepler-5 and Kepler-6 were both visited twice at each available IRAC bandpass, leading to a total 8 eclipses being secured as part of the 800 hours allocated to the program PID 60028 (PI: D. Charbonneau). We present here the first observations from this program which at characterizing and vetting Kepler candidates. Each visit is secured in full frame mode $(256 \times 256)$ with an exposure time of 10.4 seconds, at 12 second cadence, leading to 2700 frames per Kepler-5 $(\mathrm{K}=11.77) 10$ hours observations and 2150 frames per Kepler-6 (K=11.71) 8 hours observations. We perform an aperture photometry with a circular aperture of variable radius. We find that the eclipse depths and errors vary only weakly with the aperture radius for all the light-curves analyzed in this project. In the case of Kepler-5, the optimal apertures are around 5 and 2.5 pixels at 3.6 and $4.5 \mu \mathrm{m}$ respectively. We estimate the background by fitting a Gaussian to the central region of the histogram of counts from the full array. The center of the Gaussian fit is adopted as the residual background intensity. After producing the photometric time series, we use a sliding median filter to select and trim outliers greater than $3 \sigma$. This process removes measurements affected by transient hot pixels and inaccurate centroid determination. In this way, we discarded approximately $2 \%$ photometric points for all the observations. We also discarded the first half hour of observations, corresponding to around a hundred frames, which are often affected by a significant telescope jitter before stabilization.

\subsection{Occultation amplitudes and associated errors}

To measure the occultation depths and their uncertainties we model the light curves with 4 parameters: the occultation depth, $d$, the orbital semi-major axis to stellar 
radius ratio (system scale), $a / R_{\star}$, the impact parameter, $b$, and the time of mid transit, $T_{c}$. Kepler-5b and Kepler-6b have both well defined transit and stellar parameters constrained by the transits from the Kepler light-curves (Koch et al. 2010, Dunham et al. 2010). Thus, we adopt these parameters, in particular the inclination and the scale of the system, by fixing $a / R_{\star}$ and $b$ to their nominal values in our eclipsing model. Only the central time and the depth of the eclipses are allowed to vary when fitting the observations.

The Warm-Spitzer/IRAC photometry is known to be systematically affected by the so-called pixel-phase effect (see, e.g., Charbonneau et al. 2005). This effect corresponds to oscillations in the measured raw light curve with an approximate period of $\sim 70$ minutes and an amplitude of $2 \%$ peak-to-peak. This artifacts has to be corrected to properly extract the eclipse depths and errors. We performa Levenberg-Marquardt least-squares fit of the transit model to observations. The best-fit model is computed over the whole parameter space $\left(d, T_{c}, K_{i}\right)$. The baseline function described above is combined with the transit light curve function so that the fit is constrained by 8 parameters ( 2 for the transit model, 2 for the linear baseline, and 4 for the pixel phase effect). To obtain an estimate of the errors in our observations we use the Prayer Bead method (Désert et al. 2009) and derived the covariance from the residuals of the light curve.

\section{Analysis of the Kepler light curves}

The Kepler pass-band spans 437 to $897 \mathrm{~nm}$, with a central wavelength roughly equivalent to the R-band (Koch et al. 2010). The stars Kepler-5 and Kepler-6 have Kepler magnitudes $(K p)$ of 16.13 and 16.13 , reflecting the color response of the Kepler photometer (Batalha 2010, Bryson et al. 2010). The light of the companion star close-by Kepler-6 as discussed above in Sect. 2. is included in the Kepler aperture. This has the effect of diluting the depth of the eclipse signal by only a few percent as shown by Dunham et al. (2010), well below the expected precision.

We use the Kepler science data of Kepler-5 and Kepler-6 from Quarter 0 to 4 (Q0-Q4). These observations have been reduced and detrended by the Kepler pipeline - They consist of long cadence integration time (30 minutes) for Quarters 0 and 1 Caldwell et al. 2010) and short cadence (1 minute) for Quarters 2 to 4 (Jenkins et al. 2010b).

We identify and trim outliers greater than $3-\sigma$ using a sliding median filter. This process rejects a very small number of photometric measurements (less than $0.01 \%$ ) compared to the total measurement number. We fit each individual eclipses between the planetary orbital phase 0.38 to 0.62 using OCCULTSMALL as a trapezoid function (see description in Sect. 2.1) assuming constant ephemeris and null limb darkening coefficients. All the transit parameters but the occultation depths and the mid-occultation times are set fixed. A linear function of time represents the baseline. Each individual fitted light-curves is then normalized to its baseline in order to produce a set of normalized eclipse light curves.

We finally phase fold and combine all the normalize light curves to produce four photometric time series, one for each observing mode (cadence) and target. We present the normalized and folded light curves obtained at short cadence for both targets. We first search for the occultation events in the combined and normalized occultation light curves. This is done by evaluating the depths of Levenberg-Marquard fits to trapezoids with the same overall shape as the planetary transits (same impact parameters and system scales), but at different phases of the orbital period. We now estimate 
the significance of these detections by measuring the occultations depths, ephemeris and associated errors for each observing cadence mode and targets. We estimate the parameter values and errors using a bootstrap Monte-Carlo analysis.

\section{Results}

We find that decreases in the fluxes of the Kepler and Warm-Spitzer light-curves correlated with the expected phases of occultations are detected in all band-passes at nearly $3-\sigma$ level. We thus interpreted these signals as planetary occultations detected in the optical and the near infrared wavelengths.

Acknowledgements. This work is based on observations made with the Spitzer Space Telescope, which is operated by the Jet Propulsion Laboratory, California Institute of Technology, under contract to NASA. Kepler was selected as the 10th mission of the Discovery Program. Funding for this mission is provided by NASA.

\section{References}

Borucki, W. J. et al. 2009, Science, 325, 709

Bryson, S. T., et al. 2010, APJL, 713, L97

Caldwell, D. A., et al. 2010, APJL, 713, L92

Charbonneau, D., et al. 2005, APJ, 626, 523

Desert et al. 2009, APJ, 699, 478

Dunham, E. W., et al. 2010, APJL, 713, L136

Fortney, J. J. et al. 2005, APJL, 627, L69

Koch, D. G., et al. 2010, APJL, 713, L131

Latham, D. W., et al. 2010, APJL, 713, L140

Seager, et al. 2005, APJ, 632, 1122 